\title{
Induction of the Field at Different Distances from the Permanent Magnet and Between Opposing Magnets. Testing of the Superposition Principle
}

\author{
Maria Polismakova, Vera Ershova, Alexander Sandulyak, Anna Sandulyak
}

\begin{abstract}
The measurement data of the field induction at different distances from the permanent magnet Nd-Fe-B and between two equivalent magnets on distance are presented. It is shown that the obtained coordinate dependences are described by exponential function, but for a system of two magnets -only partially: up to the central area of the system and make 20-30\% of the entire area between opposing magnets. Using the example of the coordinate dependence of induction for one of two identical magnets, it is shown that the use of the superposition principle makes it possible to obtain a coordinate dependence of induction between opposing magnets, which is equivalent to experimental one.
\end{abstract}

Index Terms: permanent magnet, system of two magnets, coordinate dependence of induction, superposition principle

\section{INTRODUCTION}

The information about the characteristics of the magnetic field in the area of magnetic zone is the major information by creating laboratory and industrial devices for magnetic effects. In particular, it can be represented in the form of specially obtained coordinate dependences of the magnetic field induction $\mathrm{B}[1,2]$, i.e. data $\mathrm{B}$, depending on the coordinates of one or another point of this zone.

In a number of modern magnetic devices, for example, those intended for the separation of ferroparticles of the dispersed phase of technogenic and biological media or to control the behavior of these particles, i.e. implementation of the processes of magnetic separation (magnetophoresis), permanent magnets are used, mainly based on $\mathrm{Nd}-\mathrm{Fe}-\mathrm{B}$ [3-10]. In this case, the question of their positioning in the device for creating a working zone of magnetic influence on the medium, for example, with respect to the channel with the medium flow, is solved differently (Fig. 1a, b). Usually, this positioning is such that the working area is the area adjacent to the pole surface of the magnet, or the area located between opposing magnets.

Revised Manuscript Received on July 09, 2019.

Maria Polismakova, Vera Ershova, Alexander Sandulyak, Anna

Sandulyak, MIREA - Russian Technological University, Moscow, 119454

RussiaMoscow Polytechnic University, Moscow, 107023 Russia
In any of these (and other) cases, the coordinate dependence of the magnetic induction $\mathrm{B}$ is much demanded. Here is presented quantitative characteristic of the values of $\mathrm{B}$, which vary with distance $\mathrm{x}$ from the surface of the magnet (Fig. 1a) or from the surface of one of the opposing magnets (Fig. 1b).

In addition, if both characteristics are available, then the question of the validity of using the principle of superposition seems to be important for practical determination of the resultant values of induction between two opposing magnets.

\section{RESULT AND DISCUSSION}

Figure 2 shows the experimental coordinate dependence of the induction $B$ of the magnetic field produced by a permanent Nd-Fe-B magnet with a diameter of $25 \mathrm{~mm}$ and a thickness of $10 \mathrm{~mm}$. To perform the corresponding measurements, two equivalent (successively visible from data $B$, marked with the dots $\circ$ and $\bullet$ in Fig. 2) magnet were used in turn. Data $B$ was obtained by a Milliteslametr with Hall's sensor shifted in axial steps, normal to the surface of the magnet, in direction $x$ (Fig. 1a).

According to Fig.2, it can be seen that the coordinate dependence of induction $B$ with distance from the pole surface of the magnet decreases rapidly and at a distance commensurable with the diameter of the magnet, the values of $B$ compared with the values of $B$ at the surface of the magnet decrease more than by ten times (Fig. 2a).

The representation of this dependence in semi-logarithmic coordinates (Fig. 2b) shows that it is quasi-linearized quite well here, indicating that it correspondsto an exponential type function:

$$
B=B_{0} \exp (-k x)
$$

with the "starting" value here, $B_{0}=0.35 \mathrm{~T}$ and the coefficient in the exponent $k=110 \mathrm{~m}^{-1}$.

Fig. 3 (points $\Delta$, curves 1) shows the experimental coordinate dependences of the induction $B$ field between two equivalent (as follows from the data in Fig. 2) constant $\mathrm{Nd}-\mathrm{Fe}-\mathrm{B}$ magnets with a diameter of $25 \mathrm{~mm}$ and a thickness of $10 \mathrm{~mm}$, mutually separated on distance $b$ : from $b=13 \mathrm{~mm}$ to $b=33 \mathrm{~mm}$. As before, the values of $B$ were measured with a Milliteslametr with a Hall's sensor, shifted 


\section{Induction of the Field At Different Distances From the Permanent Magnet and Between Opposing Magnets. Testing of the Superposition Principle}

stepwise in the axial direction $x$ (Fig. 1b), normal to the surface of the magnet. These dependences (Fig. 3), obtained for a system of two opposing magnets, demonstrate the expected increase in induction values as compared to the dependence for a single magnet (Fig. 2); the degree of this increase depends on the distance $b$ between the magnets, while decreasing $b$ it grows.

Having experimental magnetic coordinates of induction $B$ of the magnetic field between two opposing equivalent magnets (Fig. 3, points $\Delta$ and approximating curves 1) and coordinate dependence of induction $B$ for one of them (Fig. 2, points $\bigcirc$ and $\bullet$, approximated by a single curve), it is of interest to check the competence of using the principle of superposition of values of $B$. This refers to obtaining the resulting coordinate dependencies $B$ on the basis of the coordinate dependence $B$ for a single magnet and information about the distance and $b$ from it in case the opposing magnet was not placed yet.

In Fig. 4, this is schematically shown by the example of the coordinate dependences of induction (similar to Fig. 2), which are mirror-like to one another, characterizing equivalent magnets located at mutual distance $b$. So, using one of the induction values from the coordinate dependence (point $B^{\prime}$ on the head section of the left coordinate dependence, that is, taken on the section up to the characteristic axis of symmetry of a two-magnet system) and the corresponding second value of induction (point $B$ "on the tail section of the right coordinate dependence), by adding these values we obtain resultant induction value (point $B=B$ $\left.+B^{\prime \prime}\right)$.

Considering the above, in Fig. 3, in addition to the real coordinate dependences of the field induction between two magnets (points $\Delta$ and curves 1 ), the experimental coordinate dependence of the field induction created by a single magnet (located below point $\circ$ and curve 2) is shown.But this dependence is represented here with an artificial "break" that falls in the middle of one or another distance $b$ between opposite magnets. Thus, within the interpolar half-space, a possible variant of visual testing of the principle of the superposition of field induction values between opposing magnets is simulated, the individual characteristics of which are known (Fig. 2).

The corresponding addition of induction values (Fig. 3: points $\circ$ along the trend of the head and tail parts of curve 2) brings to the resulting coordinate dependence: for different values of $b$, these dependences are shown in Fig. 3 with the same, but located above the points $\bigcirc$ - according to the trend of curve 1 . It can be seen that these resulting coordinate dependences of induction, obtained by superposition, are quite consistent with the dependencies obtained by direct measurements of induction between opposing magnets (points $\Delta$ and curves 1 in Fig. 3).

Moreover, such agreement (Fig. 5, points $\square$ ) is also observed for the case when direct measurements of induction between opposing magnets were carried out in the direction, as before, in the normal pole surface of the magnet, but not along the axis of the magnet, but parallel to it at a distance equal to half the radius of the magnet (Fig. 1b). At this distance, there is still no noticeable distortion of the magnetic field lines (Fig. 1b).

In Fig. 6, in semi-logarithmic coordinates, the direct field induction data between opposing magnets and the corresponding induction data obtained by superposition are described as described above, namely those contained in Fig. 3 and Fig. 5. It can be seen that these, well generalizeddata up to a certain value $x<b / 2$, namely $x=(0.7-0.8) b / 2$, are quasi-linearized, thereby obeying an exponential function of the type (1), but with individual (for each value of the distance between the magnets $b$ ) parameters $\mathrm{B}_{0}$ and $k$.

The values of the parameters $B_{0}$ and $k$ can be set by appropriate processing of the data shown in Fig.6: the results are shown in Fig.7. It is seen that for the accepted range $b=$ $13-33 \mathrm{~mm}$, or rather, for the range $b / D=0.52-1.32$, where $D$ is the magnet diameter, the parameters $B_{0}$ and $k$ can be represented by such functions from $b$ :

$$
\begin{gathered}
B_{0}=B_{x} \exp (-z \cdot b) \\
k=p \cdot b
\end{gathered}
$$

with the values of phenomenological parameters: $B_{x}=0.46$ $\mathrm{T}, z=8.3 \mathrm{~m}^{-1}, \mathrm{p}=3200 \mathrm{~m}^{-2}$.

Then, as applied to the adopted system of two opposing permanent magnets, taking into account expressions (1) (3), the expanded expression for induction between them can be written as:

$$
B=B_{x} \exp (-z b) \cdot \exp (-p b x)
$$

with the stipulated above values of the phenomenological parameters $B_{x}, z$ and $p$.

It should be noted once again that the part of the interpolararea, for which expression (4) is right, makes up $70-80 \%$ of the whole of this area (judging by the coordinate dependencies of the induction shown in Fig. 6). In the central part (up to $20-30 \%$ ), the change in induction is minimized: the field becomes close to homogeneous.

\section{CONCLUSION}

Experimental coordinate dependences of induction (at different distances from the pole surface of a permanent $\mathrm{Nd}-\mathrm{Fe}-\mathrm{B}$ magnet with a diameter of $25 \mathrm{~mm}$ and thickness of $10 \mathrm{~mm}$ ) are described. It is shown that they obey the function of an exponential form. The experimental coordinate dependences of induction between two equivalent magnets located at one or another distance are also described.It was shown that they obey the functions of the exponential form only partially: to the central area adjacent to the symmetry axis of the system and constituting 20-30\% of the entire area between the opposing magnets. The validity of the principle of superposition (using the coordinate dependence of induction for one magnet) was confirmed when it is necessary to obtain the coordinate dependence of 
induction between opposing magnets.

\section{REFERENCES}

1. A.V. Sandulyak, A.A. Sandulyak, M.N. Polismakova, D.O. Kiselev, D.A. Sandulyak, "Faraday Magnetometer With Sheric Pole Pieces: Identification Zone With A Stable Force Factor" Rossiyskiy tekhnologicheskiy zhurnal (Russian Technological Journal). 2017; 5(6): 43-54. (in Russ.)

2. A.V. Sandulyak, A.A. Sandulyak, M.N. Polismakova, D.A. Sandulyak, V.A. Ershova, "The approach to the creation and identification of the positioning zone of the sample in the Faraday magnetometer" Journal of Magnetism and Magnetic MaterialsV.469 (1) 2019, P. 665-673.

3. V. Zezulka, P. Straka, P. Mucha, "A magnetic filter with permanent magnets on the basis of rare earth" J. Magn. Magn. Mater. 268 (2004) P.219-226.

4. J.M.D. Coey, "Permanent magnet applications" J. Magn. Magn. Mater. 248 (2002) P. 441-456.

5. F. Mishima, T. Terada, Y. Akiyama, Y. Zumi, H. Okazaki, S. Nishijima, "Research and development of superconducting magnetic separation system for powdered products" IEEE Transactions on Applied Superconductivity 2008. 18 (2), art. no.4520245, P. 824-827.

6. C.M. Earhart, R.J. Wilson, R.L. White, N. Pourmand, S.X. Wang, "Microfabricated magnetic sifter for high-throughput and high-gradient magnetic separation" J. Magn. Magn. Mater. 321 (2009) P. 1436-1439.

7. J. Li, Y. Liu, S.J. Gao, M. Li, Y.Q. Wang, M.J. Tu, "Effect of process on the magnetic properties of bonded NdFeB magnet” J. Magn. Magn. Mater. 299 (2006) P. 195-204.

8. Y. Liu, H. Peng, Hu. Mingzhen, "Removing iron by magnetic separation from a potash feldspar ore" J. Wuhan Univ. Technol. Mater Sci. Ed. 28 (2) (2013) P. 362-366.

9. J. N. Darton, B. Hallmark, P. Agrawal, T. James, Vincent H.B. Ho, Nigel K.H. Slater. On the magnetic field architecture required to capture superparamagnetic nanoparticles in a microcapillary flow, J. Nanoparticle Res.12 (1) (2010) P.307-317.

Sandulyak A.V., Polismakova M.N., Sandulyak A.A., Sandulyak D.A., Ershova V.A. Convergence check of data from step-by-step experimental-computational magnetic control of iron impurities in raw materials using different field sources. Glass and Ceramics. (2018)№ 9-10(74). 332-336.

\section{FigURE CAPTIONS}

Fig.1. Visualization of the magnetic field lines in the working area: a) adjacent to the pole of permanent magnet $b$ ) between opposing unlike poles of two magnets.Here the options for the location of the working area (in particular, the channel in the flow of the medium: shown in dotted lines) for magnetic impactof medium in the working area are given; $x$ the direction of the stepwise measurement of the induction field.

Fig.2. The change in the induction of the field created by a permanent Nd-Fe-B magnet with a diameter of $25 \mathrm{~mm}$ and a thickness of $10 \mathrm{~mm}$, at the distance from the pole surface of the magnet in the axial direction; light and dark points $\circ$ and - - experimental data separately for each of the two magnets (it is quite obvious that they are equivalent); a) and b) - in usual and semi-logarithmic coordinates.

Fig.3. The change in the field induction between two equivalent opposing (at the mutual distance $b$ ) magnets with increasing of distance from the pole surface of one of the magnets in the axial direction; a) $-b=13 \mathrm{~mm}, \mathrm{~b})-b=18 \mathrm{~mm}, \mathrm{c}$ ) $-b=23 \mathrm{~mm}, \mathrm{~d})-b=29 \mathrm{~mm}, \mathrm{e})-b=33 \mathrm{~mm}$; points $\Delta$ and curves 1 are experimental data, located below points $\circ$ and curves 2 are experimental data for a single magnet taken from Fig. 2 (the corresponding dependence is presented here with a "break" that falls in the middle of the distance between the magnet and the conventionally opposing magnet: in the form

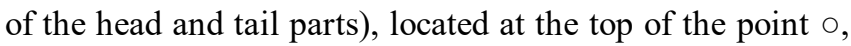
consistent with curves 1 - the data of superposition of induction on the primary data ( similar points $\circ$ on curves 2 ) for a system of two opposed magnets.

Fig.4. A schematicalimage of the decreasing coordinate dependences of induction independent created by opposing magnets located at mutual distance of $b$ and an illustration of the resulting dependency: for arbitrary private induction values, marked with $B=B^{\prime}+B^{\prime \prime}$.

Fig.5. The same as in Fig. 3, but in process of removal from a pole surface of the magnet in a direction parallel to the axial one at a distance equal to half the radius of the magnet (Fig. 1b); a) $-b=13 \mathrm{~mm}$, b) $-b=23 \mathrm{~mm}$, c) $-b=33 \mathrm{~mm}$; $\square$ experimental data.

Fig.6. The data offield induction between opposing magnets given in Fig. 3 and Fig.5 presented herein semi-logarithmic coordinates.

Fig.7. Illustration of changing the parameters $B_{0}$ and $\mathrm{k}$ depending on the mutual removal of the opposing magnets.
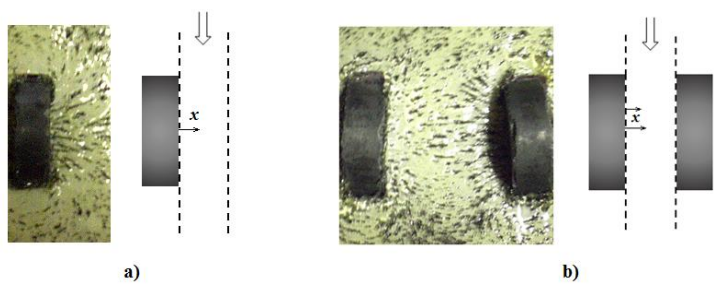

Fig.1.
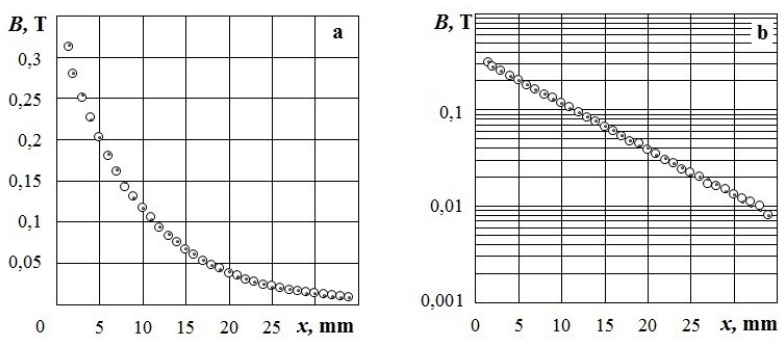

Fig.2 
Induction of the Field At Different Distances From the Permanent Magnet and Between Opposing Magnets. Testing of the Superposition Principle

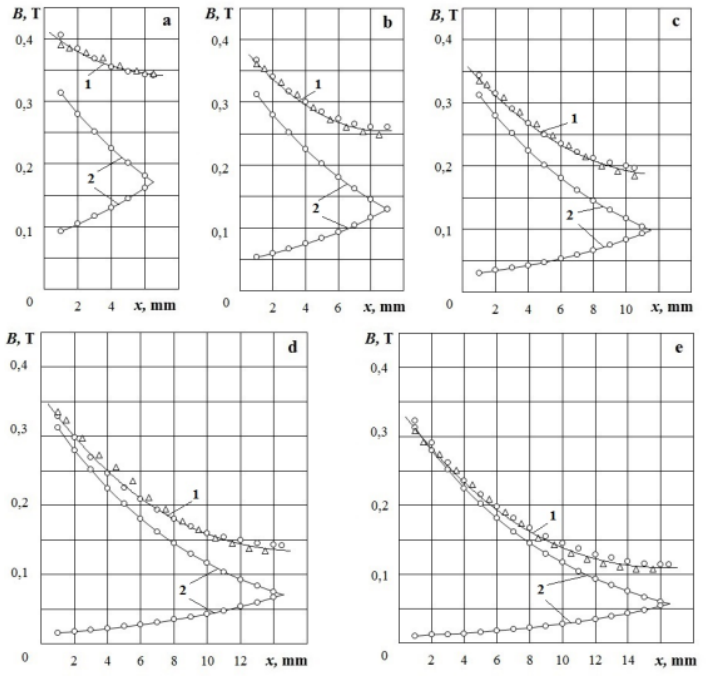

Fig.3.

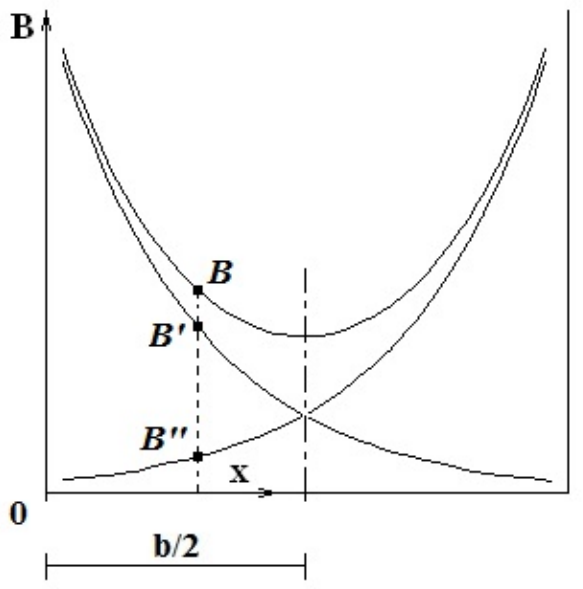

Fig.4.

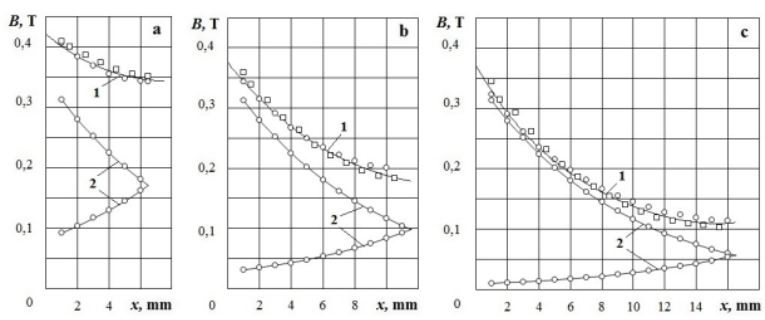

Fig.5.
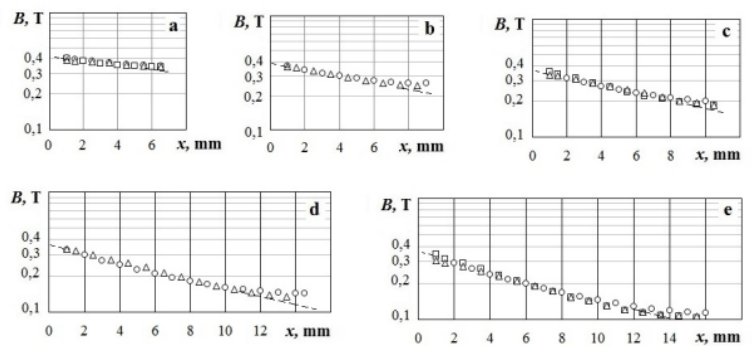

Fig.6.
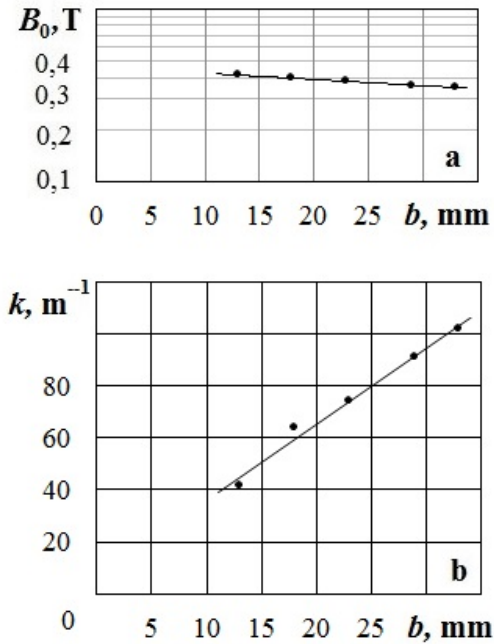

Fig.7. 